\title{
Cerebral Air Embolism: a Case Report with an Emphasis of its Pathophysiology and MRI Findings
}

Investigative Magnetic Resonance Imaging

\section{Case Report}

Received: November 15, 2018

Revised: December 5, 2018

Accepted: December 18, 2018

\section{Correspondence to:}

See Sung Choi, M.D., Ph.D. Department of Radiology, Wonkwang University Hospital, 895 Muwang-ro, Jeonlabuk-do 54538 , Korea.

Tel. +82-63-859-1920

Fax. +82-63-881-4749

E-mail: sschoi@wonkwang.ac.kr

This is an Open Access article distributed under the terms of the Creative Commons Attribution Non-Commercial License (http://creativecommons.org/licenses/ by-nc/4.0/) which permits unrestricted non-commercial use, distribution, and reproduction in any medium, provided the original work is properly cited.

Copyright (C) 2019 Korean Society of Magnetic Resonance in Medicine (KSMRM)

\author{
Se Ri Kang, See Sung Choi, Se Jeong Jeon \\ Department of Radiology, Wonkwang University Hospital, Jeonlabuk-do, Korea
}

Cerebral air embolism (CAE) is a rare complication of various medical procedures. It manifests with symptoms similar to those of typical acute cerebral infarction, however the treatment is quite different. We present a case of arterial CAE that was associated with a disconnected central venous catheter and appeared as punctate dark signal intensities with aliasing artifacts on the susceptibility-weighted filtered phase magnetic resonance image. The susceptibility-weighted filtered phase image can be helpful for diagnosing CAE and the magnetic resonance imaging reflects the pathophysiology of CAE.

Keywords: Air embolism; Intracranial embolism; Magnetic resonance imaging

\section{INTRODUCTION}

The introduction of air into cerebrovascular circulation, termed cerebral air embolism (CAE), can occur iatrogenically or spontaneously. The varied conditions associated with CAE include cardiac surgery, central venous catheter manipulation, hemodialysis, endoscopy, and laparoscopy, among others (1-3). CAE may induce variable neurologic symptoms from a sudden change in sensorium to disorientation to coma, mimicking typical acute cerebral infarction (1). However, with CAE, the acute treatment options differ from treatment of a typical ischemic stroke and the doctor should consider emergent hyperbaric oxygen therapy (4). Therefore, rapid diagnosis and management are important when encountering CAE. Several brief reports have been published presenting the magnetic resonance imaging (MRI) findings of CAE. However, to the best of our knowledge, only a few reports have correlated the pathophysiology of CAE with its imaging findings. Here, we present a case of arterial CAE associated with a disconnected central venous catheter, and review the various reports of CAE with an emphasis on the pathophysiological mechanism and MRI findings.

\section{CASE REPORT}

A 66-year-old man, who was residing in a nursing hospital after an operation for gastric cancer was admitted to our emergency department by ambulance with chief complaints of dyspnea and mental changes that occurred two hours prior. Upon 
admission, the patient's oxygen saturation was $88 \%$, but it increased to $94 \%$ after supplementation by oxygen mask. His blood pressure $(120 / 80 \mathrm{mmHg})$ and body temperature $\left(37.8^{\circ} \mathrm{C}\right)$ were normal. He was in a semi-comatose state, therefore we could not perform sensory and motor function tests. His family claimed there was no history of trauma or chronic respiratory disease, and his initial lab findings revealed increased levels of C-reactive protein $(61.68 \mathrm{mg} / \mathrm{L}$; normal range $0-5 \mathrm{mg} / \mathrm{L}$ ).

Immediately, the clinicians suspected acute cerebral infarction and conducted a brain MRI. The brain MRI revealed multifocal hyperintense lesions in the vascular watershed territories on diffusion-weighted imaging (DWI) (Fig. 1a), with an decreased apparent diffusion coefficient (ADC) value (Fig. 1b), suggesting acute cerebral infarction. All sequences, including susceptibility-weighted imaging (SWI) revealed multifocal dark signal intensities (white arrows) within the acute infarct area. These lesions presented as dark signal intensities with surrounding aliasing artifacts on the SW filtered phase image (Fig. $1 d)$. To determine if these intensities were indicative of microhemorrhage, calcification, or air, a brain computed tomography (CT) scan (Fig. 1g) was performed, which revealed multiple low attenuation lesions, suggestive of

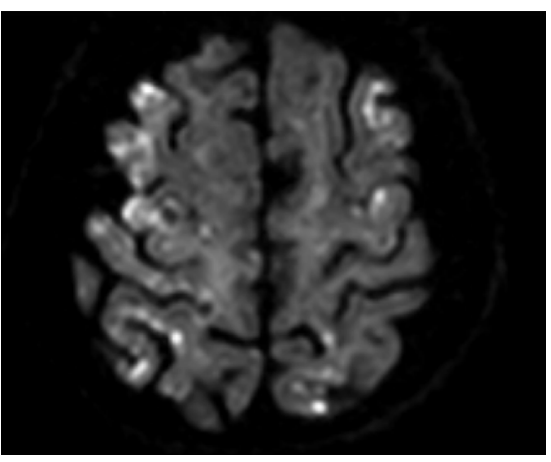

a

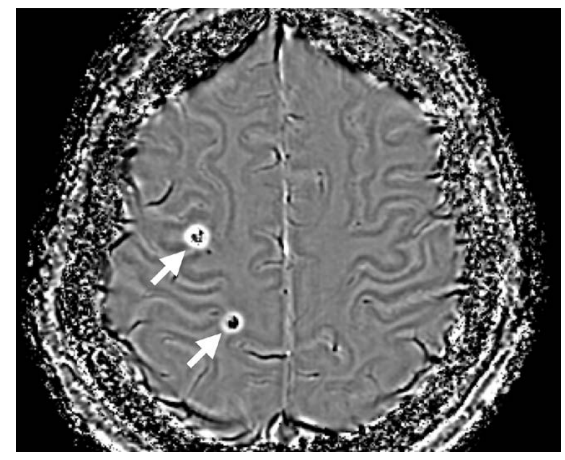

d

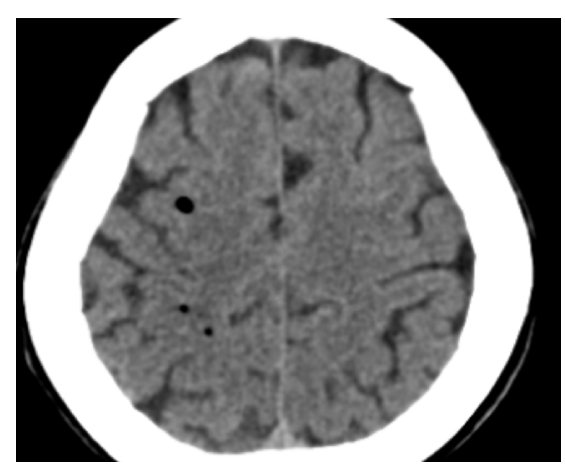

g

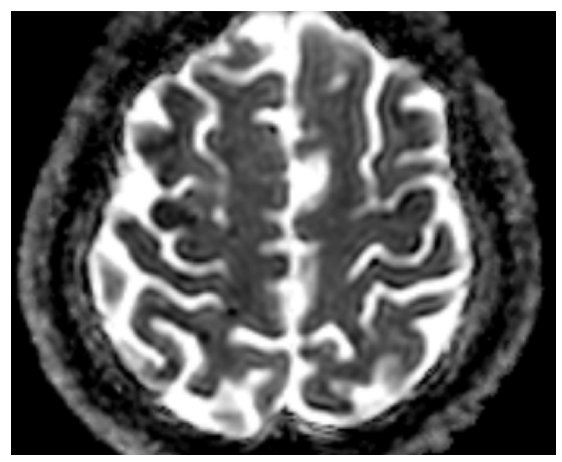

b

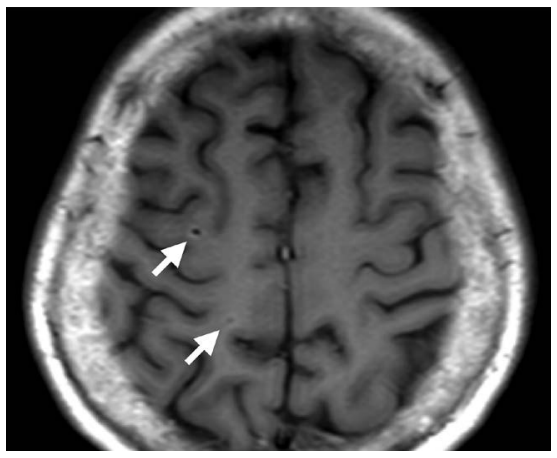

e

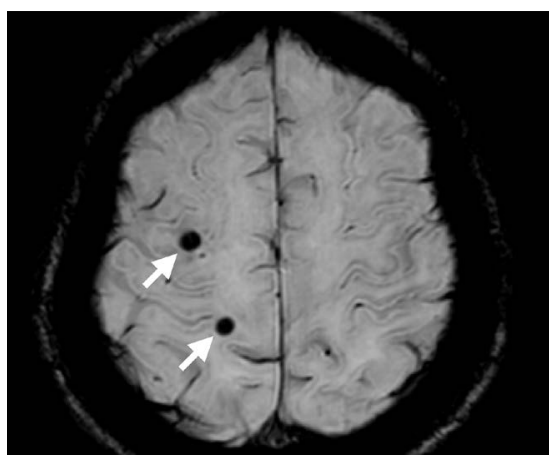

C

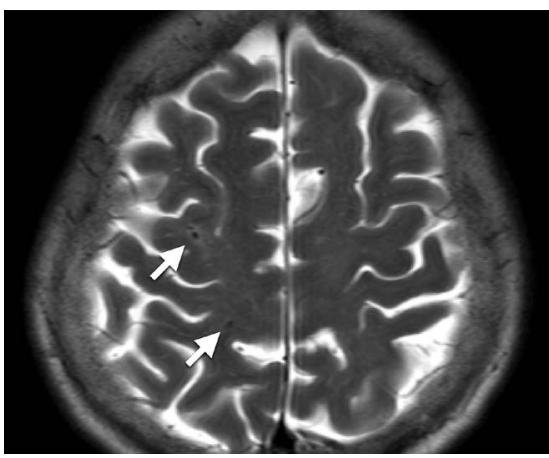

f

Fig. 1. Initial brain MRI and CT two hours after the onset of symptoms. DWI (a) demonstrates multifocal hyperintensities, seen as low signal foci on the ADC map (b), indicating acute cerebral infarction. SWI shows multifocal dark signal intensities (white arrows) on magnitude image (c) and aliasing artifacts on filtered phase image (d). They also present as dark signal intensities (white arrows) on T1-weighted image (e) and T2-weighted image ( $\mathrm{f}$ ). Immediate brain CT (g) shows multiple punctate air bubbles, indicating arterial CAE. 


\section{CAE.}

As a next step, a chest CT scan was acquired to evaluate the pulmonary air embolism, which revealed no air bubble in the pulmonary or cardiac vasculature, but pneumonia in both lungs. With a suspicion of septic infarction with gas-generating bacterial meningitis, a cerebrospinal fluid analysis was also performed, which indicated no evidence of infection.

Meanwhile, when the patient was referred to our hospital, he had a central venous catheter inserted at an outside hospital and the catheter was disconnected. So we believed he had a CAE associated with the disconnected central line. We removed the catheter and treated him with high oxygen and left lateral decubitus positioning to rescue his left hemisphere. We also performed an echocardiography to detect an intra-cardiac shunt. However, the echocardiography evaluation was limited because the patient was uncooperative, and no apparent intra-cardiac shunt was seen.

A follow-up MRI performed 12 days after admission revealed vasogenic edema in the bilateral fronto-parietal lobe and improvement of the preexisting acute cortical infarction on DWI (Fig. 2a) and ADC map (Fig. 2b). SWI (Fig. 2c, d) revealed multiple dark signal intensities which were thought to be a residual air embolism and microhemorrhage. No additional CT scan was performed as the patient became more conscious.

\section{DISCUSSION}

CAE is a potentially catastrophic event and usually results from iatrogenic causes. During manipulation of a central line or during other procedures, entry of air into the blood stream can occur when a pressure gradient exists in which the venous pressure is lower than the atmospheric pressure, for example, in deep inspiration, hypovolemia, or upright position of the patient (5). Small amounts of air introduced into the venous circulation are often tolerated, however a bolus injection of air of $>1.5-3 \mathrm{~mL} / \mathrm{kg}$ exceeds the filter capacity of the pulmonary filter and results in embolization (1). Venous CAE is thought to result from the retrograde rise of air in the systemic venous circulation, particularly in an upright position (2). However, venous air can enter into the cerebral arterial circulation when the volume of the introduced air exceeds the pulmonary filter capacity when there is a right-to-left intra-cardiac shunt, such as in cases of atrial septal defect or patent foramen ovale, and when

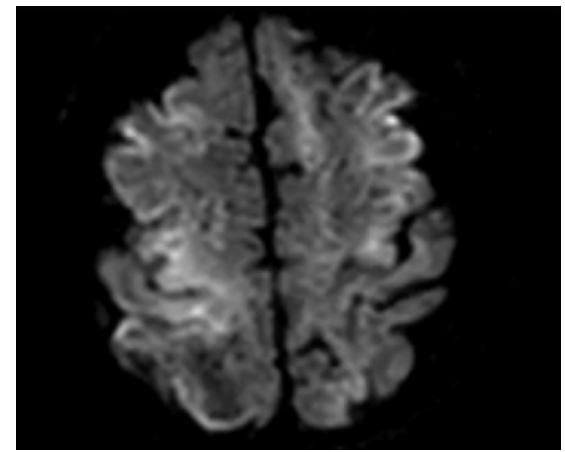

a

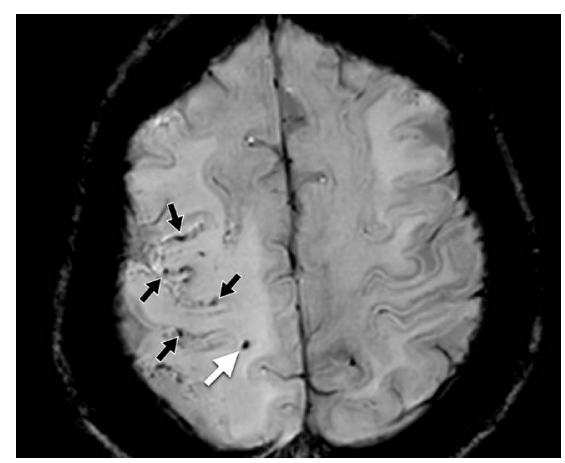

C

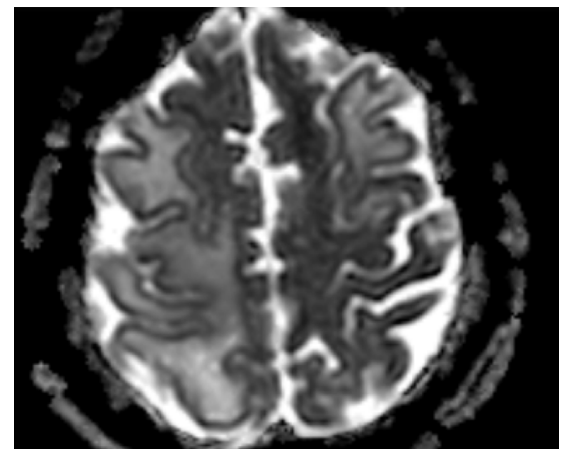

b

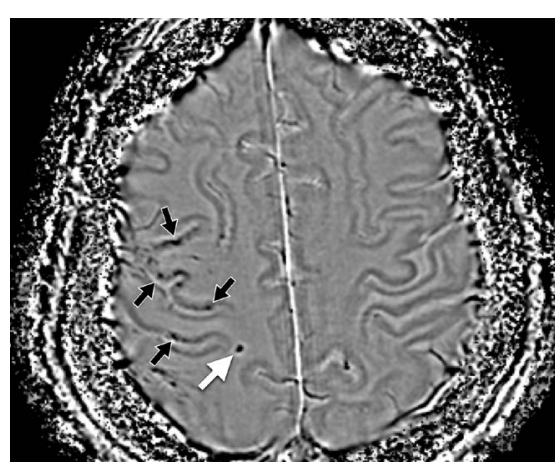

d
Fig. 2. Follow-up MRI 12 days later. DWI (a) and ADC map (b) show new diffuse progressing vasogenic edema in cerebral hemispheres. SW magnitude image (c) and filtered phase image (d) show new multifocal tiny dark signal intensities (black arrows) without blooming or aliasing artifacts, suggesting microhemorrhage. The preexisting air bubble (white arrow) shows aliasing artifacts. 
air directly enters the systemic arterial circulation, e.g., through the pulmonary vein $(1,2,6)$.

Depending on the distribution of air, CAE can be observed as two patterns. Venous CAE appears as serpiginous air densities along the cortical sulci, particularly in the frontal cortical area, with gyriform patterns of acute infarction near the air, and is thought to be related to the position of the patient at the time of air entry $(7,8)$. On the other hand, arterial CAE presents as punctate air densities in the brain parenchyma (6), such as was the case of our patient (Fig. 1c, d).

Our case presented no cardiopulmonary anomalies, such as intra-cardiac shunt or pulmonary arteriovenous malformations. However, an echocardiography was likely to miss the intra-cardiac shunt because the patient was uncooperative, and in addition, pulmonary vessels in this patient were likely to be damaged from the infection of both lungs. Therefore, in this case, a large amount of venous-originating air, introduced via the disconnected central line, is believed to have triggered arterial CAE by overcoming the lung filtration capacity and entering the cerebral arterial circulation through the missed intracardiac shunt or pneumonia-damaged pulmonary vessels.

CAE can be easily detected by its air density on CT, however we should be able to consider CAE on MRI when the MRI is performed first, especially in a case with a recent history of iatrogenic procedures, such as in our case. Air has very low magnetic susceptibility and can induce signal loss on MRI, particularly on gradient-echo MRI (9). However, intracranial air bubbles can be mistaken for blood products or cortical vessels. Hwang and Kim (9) found that venous CAE presented as engorged tubular dark signal intensities with aliasing and blooming artifacts on SWI, due to marked susceptibility artifacts caused by air. Our case also presented with ovoid dark signal intensities with aliasing artifacts on the SW filtered phase image (Fig. 1d). On follow-up images (Fig. 2c, d), the preexisting air embolism with aliasing artifacts became smaller (white arrow) and tiny dark signal intensities without artifacts, indicative of microhemorrhage, newly-appeared (black arrows). Although large hemorrhage may also show heterogeneous signal intensity and even aliasing artifacts on SW filtered phase image, acute or hyperacute hemorrhage, which could be associated with acute symptoms in our patient, do not present as dark signal intensities both on T1-and T2-weighted images, as seen in our patient's images (Fig. 1e, f). Therefore, from this case we learned that we should consider CAE when encountering dark signal intensities on all MR sequences with aliasing artifacts on the SW filtered phase image, and that this consideration may lead to rapid diagnosis and treatment.

Finally, lodged air emboli in the smaller arteries of the brain obstruct the bloodstream and result in hypoxia, ischemia, and cerebral edema (1). The air bubble may act as a foreign body, producing an inflammatory reaction and directly damaging the endothelial cells of the vessel wall $(1,5)$. Therefore, diffuse vasogenic cerebral edema which appeared on follow-up MR images (Fig. 2a, b) of this patient was believed to be extracellular fluid leakage from the capillaries due to recruitment of the inflammatory cytokines and associated breakdown of the blood-brain barrier. Also, microhemorrhage seen on follow-up SWI (black arrows in Fig. 2c, d) was thought to be due to the damage of the vessel wall by the air bubble, as mentioned above.

The treatment of CAE starts with identification of the source of air entry and avoidance of further air embolization (10). Emergent treatment includes administration of $100 \%$ oxygen, transport of the patient to a hyperbaric facility, and a bolus dose $(10 \mathrm{mg})$ of dexamethasone to prevent cerebral edema (1). Hypothermia can also be considered as a neuroprotective approach (3). Among them, hyperbaric oxygen therapy is important because its high pressure reduces the volume of the air embolus and hyperoxygenation facilitates gas removal via denitrogenation, thereby maintaining oxygenation in the ischemic tissues (8). However, in our case, hyperbaric oxygen therapy could not be applied because the patient was so unstable that he could not be moved to a suitable facility.

In conclusion, CAE should be diagnosed early and differentiated from typical acute cerebral infarction which can manifest with similar symptoms, and from hemorrhage, which also presents dark signal intensities and even aliasing artifacts on SWI. Integrated interpretation considering imaging findings on SW filtered phase images and T1- and T2-weighted images can be helpful to differentiate $C A E$, and MRI findings are useful to identify and understand the pathophysiology of CAE.

\section{REFERENCES}

1. Jain KK. Textbook of hyperbaric medicine. 6th ed. Switzerland: Springer, 2017:121-129

2. Mishra R, Reddy $P$, Khaja M. Fatal cerebral air embolism: a case series and literature review. Case Rep Crit Care 
2016;2016:3425321

3. Bauerle J, Fischer A, Hornig T, Egger K, Wengenmayer T, Bardutzky J. Therapeutic hypothermia in cerebral air embolism: a case report. Springerplus 2013;2:411

4. Lin C, Barrio GA, Hurwitz LM, Kranz PG. Cerebral air embolism from angioinvasive cavitary aspergillosis. Case Rep Neurol Med 2014;2014:406106

5. Suri V, Gupta R, Sharma G, Suri K. An unusual cause of ischemic stroke - Cerebral air embolism. Ann Indian Acad Neurol 2014;17:89-91

6. Togo M, Hoshi T, Matsuoka R, Imai Y, Kohara N. Multiple small hemorrhagic infarcts in cerebral air embolism: a case report. BMC Res Notes 2017;10:599

7. Caulfield AF, Lansberg MG, Marks MP, Albers GW, Wijman
CA. MRI characteristics of cerebral air embolism from a venous source. Neurology 2006;66:945-946

8. Kaichi $Y$, Kakeda $S$, Korogi $Y$, et al. Changes over time in intracranial air in patients with cerebral air embolism: radiological study in two cases. Case Rep Neurol Med 2015;2015:491017

9. Hwang Y, Kim YJ. Retrograde cerebral venous air embolism on susceptibility-weighted imaging. Can J Neurol Sci 2018;45:464-465

10. Han SS, Kim SS, Hong HP, Lee SY, Lee SJ, Lee BK. Massive paradoxical air embolism in brain occurring after central venous catheterization: a case report. J Korean Med Sci 2010;25:1536-1538 